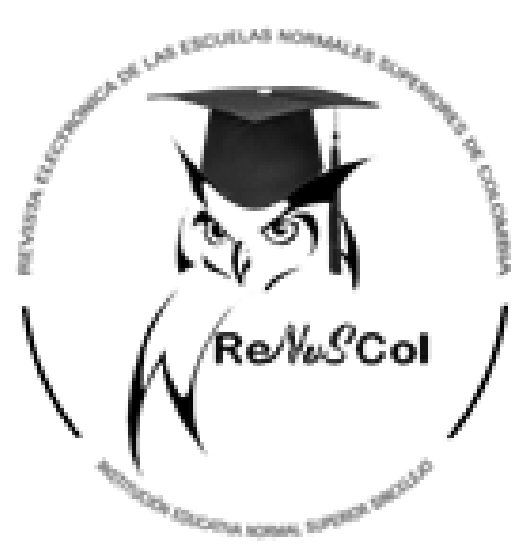

Autor(es):

PhD. Octavio Carlos Rodríguez Tovar

ORCID: 0000-0002-2498-6172

Docente Investigador Institución Educativa Normal Superior de Corozal

Email: octaviort73@gmail.com

Cómo citar este texto:

Rodríguez Tovar O. C. (2021). Análisis epistemológico de la obra pedagógica del oprimido de paulo freire: un camino delibertad. ReNosCol. No. I, Vol I. Enero-Junio/ 2021. Pp. 98-108. Institución Educativa Normal Superior Sincelejo. URLdisponible en: http://www.eumed.net/rev/renoscol.html

Recibido: noviembre 2020. Aceptado: abril de 2021. Publicado: junio de 2021.

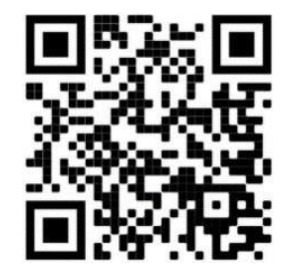




\section{Título: ANÁLISIS EPISTEMOLÓGICO DE LA OBRA PEDAGOGÍA DEL OPRIMIDO DE PAULO FREIRE: UN CAMINO DE LIBERTAD}

Resumen: Paulo Freire se ha convertido en uno de los referentes latinoamericanos más importantes, en el campo pedagógico y educativo, puesto que rompió con lógicas paradigmáticas tradicionales educativas en cuanto a las herramientas de enseñanza y aprendizaje de los procesos lectoescriturales, planteando dinámicas pedagógicas enfocadas al acercamiento contextual en que están sumergidos los sujetos que aprenden, por lo que la propuesta pedagógica se enfoca al análisis de los entretejidos socioculturales y socioeconómicos en que se movilizan, de tal manera que al comprender sus criterios geo socio-culturales, se diseñen los procesos pedagógicos a orientar. En este sentido las producciones pedagógicas de Paulo Freire son grandes fuentes documentales, para conocer su legado pedagógico, que, al acercarse a ellos, se descubren miradas y planteamientos epistemológicos, gnoseológicos y ontológicos, que se convierten, a su vez en fuentes de análisis y reflexión por distintas tendencias epistémicas y filosóficas, de tal manera que permitan identificar las tesis científicas que subyacen en ellas y someterlas a lógicas de reflexiones y análisis.

Palabras clave: Epistemología, análisis, Paulo Freire, Pedagogía, Ciencia, Educación, sociales, realidad, cultura

\section{Title: EPISTEMOLOGICAL ANALYSIS OF PAULO FREIRE'S PEDAGOGY OF THE OPPRESSED: A WAY TO FREEDOM}

Summary: Paulo Freire has become one of the most important Latin American referents, in the pedagogical and educational field, since he broke with traditional educational paradigmatic logics in terms of teaching and learning tools of the literacy processes, It proposes pedagogical dynamics focused on the contextual approach in which the learning subjects are immersed, so that the pedagogical proposal focuses on the analysis of the socio-cultural and socioeconomic interweavings in which they are mobilized, so that by understanding their geo-socio-cultural criteria, the pedagogical processes to be oriented are designed. In this sense, Paulo Freire's pedagogical productions are great documentary sources for learning about his pedagogical legacy, which, when approached, reveal epistemological, gnoseological and ontological views and approaches, which in turn become sources of analysis and reflection for different epistemic and philosophical trends, in such a way as to identify the scientific theses that underlie them and submit them to the logic of reflection and analysis.

Key words: Epistemology, analysis, Paulo Freire, Pedagogy, Science, Education, social, reality, culture.

\section{Título: ANÁLISE EPISTEMOLÓGICA DA PEDAGOGIA DE PAULO FREIRE DO OPRIMADO: UM CAMINHO PARA A LIBERDADE}

Abstrato: Paulo Freire tornou-se uma das mais importantes referências latino-americanas, no campo pedagógico e educativo, uma vez que rompeu com as lógicas paradigmáticas educativas tradicionais em termos de instrumentos de ensino e aprendizagem dos processos de leitura e escrita, A proposta pedagógica centra-se na análise dos entrelaçamentos socioculturais e sócio-económicos em que estão imersos, de tal forma que, através da compreensão dos seus critérios geo-socio-culturais, os processos pedagógicos a serem orientados podem ser concebidos. Neste sentido, as produções pedagógicas de Paulo Freire são grandes fontes documentais para a aprendizagem do seu legado pedagógico, que, quando abordadas, revelam visões e abordagens epistemológicas, gnoseológicas e ontológicas, que por sua vez se tornam fontes de análise e reflexão para diferentes tendências epistémicas e filosóficas, de modo a identificar as teses científicas que lhes estão subjacentes e submetê-las à lógica da reflexão e da análise. Palavras-chave: Epistemologia, análise, Paulo Freire, Pedagogia, Ciência, Educação, social, realidade, cultura.

\section{Introducción}

Freire desde la Pedagogía del oprimido (Freire, P. 1993) se convierte en el símbolo de reflexión y de autorreflexión de las estructuras impositivas y dogmáticas de las fuerzas de poder que han ejercido dominio político, socioeconómico e inclusive hasta el ideológico, en la población menos favorecidas y vulnerables de una sociedad, las cuales por motivos netamente económicos 
son sometidos a una serie de vulnerabilidad de sus derechos como seres humanos, como la coerción de su libre expresión y por ende de sus libertades individuales.

En este orden de ideas desde la pedagogía del oprimido se construyen estructuras educativas revolucionarias que condujeron a la movilización de una serie de dinámicas epistémicas, ontológicas, antropológicas que permitieron el cimiento de los aspectos principios: el primero el surgimiento de una nueva pedagogía basada en la comprensión de las coordenadas geosociopolíticas en que se encuentran sumergidos los sujetos que aprenden; en segundo lugar, el análisis del papel y el rol del docente como agente de cambio y transformación, el tercero el diseño de criterios didácticos que propiciaron una metodología basada en la actividad dialógica, y por último la creación de una conciencia crítica que irrumpió en un nuevo paradigma emancipatorio.

Desde inicio la construcción de sus planteamientos pedagógicos, a partir de la comprensión de las estructuras socioculturales en que se movilizan los sujetos que aprenden, para ello, leer las realidades del contexto, constituye un aspecto fundamental para generar los procesos pedagógicos que emprende no solo el docente, sino, la institución educativa, puesto que permite en primer lugar la reflexión de las coordenadas geopolítica en que se encuentra la comunidad, en segundo lugar comprender los principios plurietnoculturales, como aspecto esencial para conocer su identidad, y por último, analizar las necesidades, problemas y dificultades sociales, que se suscitan en las interacciones humanas.

Comprender las coordenadas geopolíticas, es un aspecto importante en el desarrollo de los procesos pedagógicos, puesto que estudiar el escenario geo-histórico-político, puesto que el análisis de la memoria histórica y la tradición crítica, permite conocer los procesos ideológicos que se construyen basados en procesos epistémicos que dejaron los grupos sociales que tuvieron su asiento en el territorio donde se levanta la comunidad, estos criterios ideológicos son determinativos en la construcción de los principios de organización social y política, sino también de los cimientos del establecimiento de identidad sociocultural que losrepresenta.

Por consiguiente, desde la perspectiva de la pedagogía freireana, el análisis de contexto sociocultural juega un papel fundamental en el desarrollo de la construcción de dinámicas pedagógicas, puesto que entender las tradiciones que los representan están permiten contextualizar el saber y los conocimientos a orientar durante el desarrollo de los procesos de enseñanza aprendizaje, puesto que las tradiciones simbolizan su actuar, sus actitudes, sus comportamientos, los cuales así mismo, conllevan a cimentar los principios socio-políticos que permiten la organización de la comunidad, de igual manera, generan los principios educacionales, en cuanto a que permiten definir los propósitos y los ideales de aprendizaje.

En el desarrollo de la comprensión de los procesos culturales del contexto las costumbres generadas desde las tradiciones son esenciales en el conocimiento de la dinámica pedagógica, puesto que las costumbres evidencian las tradiciones culturales que los identifican; para ello, es importante porque nos permite entender que la multidiversidad que emergen de las 
diferencias de las situaciones y contextos sociales generan contextualizar los conocimientos a mediar, definiendo para ello, no solo los perfiles educativos, sino todo un proceso de conservación y preservación de los principios costumbristas que lo identifican, por lo que la escuela debe no solo propiciar, sino, incluir en sus movilidades pedagógicas.

Por lo anterior se generó consciencia critica desde la perspectiva de la reflexión del contexto, porque las circunstancias socio-económicas y pluri-étnico culturales que suceden en la realidad son sujetos y objetos de estudios y de análisis; en este sentido las creencias se convierten en uno de los aspectos fundamentales en la reflexión de los grupos sociales, puesto que contribuye a cimentar los principios esenciales para poder construir el legado de tradiciones y costumbres que los identifican, así mismo, las creencias también generan los criterios básicos para perfilar las actitudes y los comportamientos que representan al grupo social.

Tanto las tradiciones, como las costumbres y las creencias constituyen los componentes esenciales en la construcción del legado cultural en cada uno de los grupos sociales objetos de intervención pedagógica, estos aspectos, así mismo, son estudiados desde la concepción étnica al cual pertenecen los grupos sociales, puesto que, a partir de ello, se estructuran las concepciones culturales que los simbolizan, es así como dentro de la triangulación tradiciones, costumbres y creencias emergen las miradas internas que son consolidadas desde cimientos epistémicos, antropológicos y ontológicos que sin duda trazan la hoja de ruta de su devenir.

En este orden de ideas desde el reconocimiento del legado cultural y el análisis y reflexión de los componentes que lo estructuran, puesto que se convierten en el cimiento esencial para generar los procesos pedagógicos, porque permiten no solo la contextualización de los ejes temáticos que estructuran una dinámica del conocimiento a mediar y facilitar, sino, la reconceptualización de los objetos de conocimientos a orientar. En este sentido la movilización didáctica y metodológica visibilizada por las estrategias, actividades y herramientas de aprendizaje, las cuales, al igual que los procesos evaluativos emergen de los principios y criterios identificados desde la reflexión investigativa e indagativa dada desde la lectura del contexto.

Freire generó consciencia crítica en la comunidad del conocimiento, acerca de la importancia de analizar las coordenadas que se suscitan en la realidad en que se encuentran inmersos los sujetos que aprenden, como aspecto esencial en el conocimiento de las latitudes en que interactúan los seres humanos, identificando en ellos, sus necesidades, problemas, dificultades que se presentan en el quehacer cotidiano. Estos ejes problémicos emergen de las profundas miradas internas de los acontecimientos y sucesos que transitan en las dificultades de índole económica, sociales, políticas, religiosas, y de discriminación éticas que son fundamentales estudiar, porque se inciden en los procesos pedagógicos.

Analizar los procesos sociales que emergen en las realidades de los contextos son esenciales para construir las estructuras pedagógicas son fundamentales puesto que generan el diseño de plataformas curriculares, didácticas y metodológicas para 
desarrollar un proceso educativo contextualizado y reconceptualizado que surgen del análisis social de la comunidad, dentro de esta dinámica se encuentran los grandes problemas estructurales como situaciones laborales en que se movilizan los integrantes de la comunidad, en el entendido que a partir de su desempeño laborar, se establecen los mecanismos de organización social y de orden jerárquico que son motivos de análisis en la concepción de una pedagogía social y humana.

En este sentido, las necesidades en los servicios públicos es otro de los aspectos esenciales, en la reflexión del contexto en que interactúan los participantes de una comunidad, puesto que permiten entender las grandes dificultades en que se encuentran inmersos, las cuales sus causas de origen, obedecen más que todo, en primer lugar a las grandes brechas de desigualdad social, que existen entre aquellos grupos sociales que no tienen oportunidades para acceder a mejores de condiciones de vida, desarrollan sus escenarios cotidianos en espacios inhóspitos que no favorecen la generación de principios humanos y en segundo lugar eldesequilibrio económico entre las distintos grupos sociales, conlleva a vivir en condiciones desfavorables e infrahumanas, estos son objeto de analizar, en el desarrollo de la estructuración de los procesos pedagógicos.

Por consiguiente y ante lo anterior, la comprensión de los procesos económicos, que subyacen de las dinámicas de interacción cotidianas, juegan un papel fundamental en el desarrollo de la estructuración de los procesos pedagógicos, en el sentido de reflexionar acerca de los componentes sociológicos que subyacen en la movilización de las interacciones de los grupos sociales, que sin duda inciden en las movilidades socioeconómicas, que emergen en las miradas de interacción de las comunidades sociales, para ello, es interesante que las actividades que realizan para obtener ingresos económicos dependen en gran medida de nivel educativo, de la generación de principios culturales visibilizados en las tradiciones, costumbres y creencias.

Desde esta perspectiva el análisis de las estructuras socioeconómicas que definen la movilidad en un grupo social, se cimientan desde principios sociológicos, antropológicos y etnográficos, puesto que determinan las estructuras de producción e intercambio económico; en este sentido, desde la concepción antropológica se erigen la constitución de un ser humano que comprende que su labor está orientada hacia la generación de elementos que permiten entender las dinámicas geográficas como aspecto importante para propiciar los procesos de producción desde distintas actividades que le permitirán la dinámica económica y de esta manera propiciar el mejoramiento cualitativo de las condiciones de supervivencia.

En este sentido, desde la constitución antropológica, se determinan los principios pragmáticos que permiten emprender la configuración de criterios que cimientan hechos, sucesos, acontecimientos, que propician la actuación del ser humano en el desarrollo de los procesos económicos, que a su vez dan surgimiento a los diferentes niveles sociales, de acuerdo a los ingresos económicos que poseen y a las producciones que generan; estos aspectos permiten reflexionar acerca de las incidencias de los cimientos económicos en la construcción de dinámicas de los procesos pedagógicos, puesto que contribuyen no solo a contextualizar los ejes temáticos a orientar, sino al diseño de herramientas metodológicas y didácticas que se convierten en componentes 
mediadores y facilitadores del aprendizaje.

En este sentido, dentro de los sustratos culturales que identifican al grupo social, emergen corrientes ideológicas que subyacen de las dinámicas de interacción que se realizan entre cada uno de los componentes que constituyen los grupos sociales, los cuales, a su vez cimientan corrientes políticas que permiten generar distintas expresiones y manifestaciones que sin duda alguna corresponden a las diferentes formas de pensamiento suscitadas en dar respuestas no solo a las dificultades y necesidades que surgen del actuar cotidiano, sino de los iniciativas que se emprenden del devenir histórico y social que surgen de los entretejidos sociales.

Las corrientes políticas que subyacen en las dinámicas geosociales son esenciales en el desarrollo de construcciónde la configuración antropológica y ontológica, puesto que determinan las líneas de pensamiento que emergen de los procesos de manifestaciones y expresiones de acuerdo a no solo a las posiciones y miradas a cada uno de los componentes que constituyen las dinámicas que transitan en los grupos sociales, las cuales se presentan en los distintos escenarios de participación ciudadana, que sin duda alguna fortalece los procesos de convivencia ciudadana, del análisis de estos planteamientos, surgen los principios y criterios que cimientan los procesos sociológicos, epistémicos, gnoseológicos, filosóficos y humanísticos.

Desde la perspectiva de la Pedagogía del Oprimido y dentro de la dinámica de las concepciones ideológicas que emergen de las intersubjetividades humanas, surgen distintas tendencias epistémicas enfocadas a cimentar estructuras de pensamiento que conlleven a la movilización de diferentes corrientes que desde la concepciones antropológicas y etnográficas se erige una nueva narrativa etnoepistemológica, encaminada a la comprensión de la multidiversidad de sustratos culturales determinan la constitución de diferentes variantes ideológicas que se conjugan entre sí como mecanismo de interacción humana .

Los planteamientos anteriores son fundamentales en el desarrollo de la construcción de los procesos pedagógicos, puesto que genera el diseño de las estructuras didácticas, metodológicas, curriculares e investigativas cimentadas en principios antropológicos que bajo la mirada de la epistemología humanista, se valore la condición humana como criterio fundamental en el desarrollo de los procesos educativos, propiciando de esta manera la conciencia crítica como aspecto que permite el análisis de las significaciones que se producen de las intersubtividades de las realidades humanas.

La pedagogía del oprimido invita al maestro a generar procesos de cambios y de transformaciones. No solo en los procesos pedagógicos, ni el papel que debe asumir como un servidor social, sino, en la práctica pedagógica que ejercitan a diario en el marco de su quehacer educativo, permitiendo de esta manera la generación de una nueva perspectiva de visionar el rol del docente en las dinámicas pedagógicas que transitan en el campo educativo; de igual manera se propicia la comprensión de promover un nuevo lenguaje pedagógico, enfocado a la construcción de escenarios educativos acorde con las movilizaciones que seentretejen en mundo postmoderno. 
En este orden de ideas, desde la Pedagogía del oprimido generan el diseño de estructuras curriculares que a su vez permiten el diseño de didácticas y metodológicas enfocadas a la resignificación y al replanteamiento de las lógicas instrumentalistas, reduccionistas, simplistas en que se encuentran ancladas los procesos pedagógicos tradicionales, propiciando ante ello, el planteamiento de dispositivos que conciba el proceso pedagógico de manera interactiva, donde la comprensión del contexto geopolítico en que se desenvuelven los sujetos, el análisis de las dimensiones humanas en sus procesos biopsicosociales, al igual que la construcción de didácticas cimentadas en la reflexión de la concepción multireferencial y multidimensional en que se movilizan las realidades postmodernas.

Ante lo anterior, desde la pedagogía del oprimido, se construyen las estructuras pedagógicas cimentadas en planteamientos eminentemente humanísticos y sociales; que orientan la concepción de principios curriculares, didácticos, metodológicos, evaluativos e investigativos, los cuales permitirán a su vez el diseño de dispositivos metodológicos visibilizados en técnicas y en herramientas de aprendizaje. Desde la mirada humanística se erige una visión biopsicoantropológicos.

El anterior planteamiento traza la hoja de ruta del pensamiento pedagógico freireano, puesto que conllevan a proponer postulados y principios pedagógicos desde una perspectiva humanista, la cual se enfoca a comprender las dimensiones del ser humano en su condición de sujeto y objeto de ser estudiado desde posiciones antropológicas yontológicas, en este sentido la pedagogía humanista cobra un valor esencial, puesto que irrumpen en las miradas psicológicas enfocadas a estudiar los procesos de comportamiento y actitudes ante situaciones geosociales; en las posiciones sociológicas orientadas a comprender las coordenadas geosociopolíticas en que se encuentran inmersasy la mirada axiológicas centradas en propiciar principios de convivencia e interacciones interhumanas.

Desde esta perspectiva la pedagogía centra su atención en el diseño de un currículo y de una didáctica orientada a propiciar las significaciones humanas que emergen de sus miradas introspectivas y de las intersubjetividades que surgen de las dinámicas de las relaciones e interacciones con los demás sujetos que aprenden, en este sentido un currículo cimentado en propiciar la valoración del ser humano como aspecto esencial en la orientación de los procesos de enseñanza aprendizaje demanda de políticas institucionales y de acciones pedagógicas orientadas a la comprensión humana. En este orden de ideas, el maestro juega un papel importante en el desarrollo de los procesos pedagógicos planteados desde Freire porque trasciende el rol del docente transmisor de conocimientos y de saberes para emprender procesos pedagógicos cimentados en la orientación, en la facilitación, acompañamiento y mediación del conocimiento, permitiendo de esta manera que los procesos pedagógicos se visionen desde la perspectiva de la generación de posibilidades de escenarios de participación entre los sujetos que aprenden, donde la expresión de los puntos de vista, los criterios personales y la aceptación de las diferencias, determinan una pedagogía basada en principios netamente interactiva recíproca y constructiva. 
Por consiguiente, el papel del maestro se orienta al desarrollo de principios eminentemente dialéctico, donde el diálogo de saberes se convierte en la esencia del proceso pedagógico, por lo tanto, la propiciación de escenarios de discusión de ideas la confrontación de criterios personales, la apertura de escenarios donde se debatan las coordenadas de los problemas existenciales del ser humano, las necesidades y dificultades que se suscitan en las interacciones de la realidad, son motivos de análisis y reflexión colectiva para generar procesos de construcción de planteamientos sociológicos, antropológicos y humanísticos que permitan el diseño de iniciativas pedagógicas centradas en la atención de las significaciones que se entretejen en el contexto geosociopolítico.

Ante lo anterior, se exhorta a un maestro que lidere procesos de transformación en cada uno de los contextos socioculturales, en que se encuentran inmersos, para ello, es fundamental tener en cuenta el fomento de un trabajo cooperativo, en equipo y colaborativo que propicie en él, el desarrollo de las resignificaciones continuas de los procesos pedagógicos, en el entendido, que a partir del diseño de principios didácticos se generan procesos de religajes, sinergias y articulaciones en la integración del conocimiento, así mismo, de las miradas internas del desarrollo de su práctica pedagógica, permitiendo que a partir de la reflexión y la autorreflexión de su quehacer educativo, construya el saber pedagógico.

En este sentido, al permitir el desarrollo de propiciar la reflexión y la autorreflexión de las prácticas pedagógicas durante la dinamización y movilización de los procesos de enseñanza aprendizaje, genera en el maestro actitudes autocríticas, enfocadas al análisis de cada uno de los componentes que intervienen en la interacción pedagógica, los cuales son sistematizados mediante el registro constante y permanente de los acontecimientos que ocurren en cada momento didáctico, permitiendo de esta manera cimentar el espíritu investigativo en los maestros, en cuanto a explorar si los objetos de conocimientos que orientan son pertinentes y contextualizados a los intereses y necesidades de los sujetos que aprenden, de igual modo, indagar y explorar por qué y para qué son relevantes los ejes temáticos orientados en los educandos; estos aspectos permiten que el docente realice una mirada introspectiva de su quehacer educativo.

La investigación de su práctica pedagógica, conmina al maestro a desarrollar constante y continuamente la resignificación y el replanteamiento de su quehacer educativo, a partir de una mirada introspectiva de los componentes pedagógicos, en lo referido al desarrollo curricular, metodológico, didáctico y evaluativo, que se convierten como eje mediador para desarrollar los procesos de interacción con los sujetos que aprenden, estos procesos permiten la construcción del saber pedagógico, a partir de las dinámicas de reflexión y autorreflexión, y de la sistematización de las mismas, se propicia la liberación de aquellos dogmas y principios pertenecientes a corrientes ajenos a su movilización contextual.

\section{Consideraciones Finales}

En este orden de ideas, al emanciparse el maestro se desprende de los dogmas que lo amarran, inicia el proceso de generar escenarios de interacción significativa en cuanto a la exposición de estructuras pedagógicas surgidas de la reflexión y 
autorreflexión de su práctica pedagógica, por lo que las orientaciones de los procesos diseñados se dinamizan bajo criterios eminentemente contextualizados, no solo a la luz de propiciación de los objetos de conocimientos, sino, la construcción de una didáctica que emergen de los intereses, expectativas, necesidades y visiones de los sujetos que aprenden, los cuales son producto de la profunda autocritica de los componentes y elementos que transitan en los procesos pedagógicos.

La emancipación como principio fundamental para propiciar los procesos de generación de libertades humanas, es uno de los ejes esenciales de la pedagogía freireana, puesto que determina, salir de los dogmas que imponen fuentes epistémicas e ideológicas que transitan en las movilidades de los componentes de la sociedad. La emancipación en este sentido se enfoca a construir procesos epistémicos, antropológicos, ontológicos y sociológicos, a partir de las coordenadas geosociopolíticas que se dinamizan en las realidades donde emergen los sujetos; analizando de esta manera los entramados de las significaciones que se entretejen en las interacciones humanas, en este escenario la emancipación emergen de los significados intersubjetivos.

\section{Referencias Bibliográficas}

Arellano Hernández Antonio. (2014). Epistemología Antropológica como conocimiento del hombre: el papel de la Antropología tecnociencia. Acta Sociológica. Recuperado de: https://www.sciencedirect.com/science/article/pii/S0186602814704748

Avelar Pereyra Nora. (2008). Paulo Freire: gusto por la libertad y pasión por la justicia. Caracas. Revista Educere. http://ve.scielo.org/scielo.php?script=sci_arttext\&pid=S1316-49102005000200003

Bárcenas Ramón (2012). La confrontación de ontología y Epistemología en el pensamiento gadameriano. Revista Valenciana. Guanajuato. $\quad$ http://www.scielo.org.mx/scielo.php?script=sci_arttext\&pid=S2007$\underline{5382012000100001}$

Fair. Hernán. (2014). La Teoría Crítica de Adorno y el psicoanálisis lacaniano como filosofías de la negación ontológica. Apuntes para una crítica sociocultural, epistemológica y ético-política a los valores hegemónicos del capitalismo neoliberal. San Luis Argentina: revista fundamentos en humanidades.: https://www.redalyc.org/pdf/184/18440029002.pdf

Fiori Ernani María. (2012). Aprender decir la palabra. Freirepedia Recuperado en: file:///F:/PEDAGOG\%C3\%8DA\%20DEL\%200PRIMIDO\%20PAULO\%20FREIRE.pdf

Freire, P. (1993). Pedagogía de la esperanza: un reencuentro con la pedagogía del oprimido. Siglo xxi.

Gómez Muleth Claudia. (2017). El existencialismo es un Humanismo de Jean- Paul Sartre, Bogotá. Cultura Genial. https://www.culturagenial.com/es/el-existencialismo-es-un-humanismo-jean-paul-sartre/

González Rey Fernando. (2018). Epistemología y Ontología, un debate necesario en la psicología de hoy. Scielo. Brasilia. http://www.scielo.org.co/pdf/dpp/v5n2/v5n2a02.pdf

Hincapié Juan Pablo. (2016). Elementos ontológicos, epistemológicos y metodológicos para la construcción de un marco teórico de estudio de los activos intangibles. Universidad Javeriana. Bogotá. http://www.scielo.org.co/pdf/cuco/v18n45/0123-1472-cuco-18-45-00086.pdf

Jáuregui Inmaculada. (2001). Cuestiones Epistemológicas en Antropología. Murcia Gazeta de Antropología. 
https://www.ugr.es/ pwlac/G17_16Inmaculada_Jauregui_Balenciaga.html

Mejía Correa Adriana María (2009). La investigación en ciencias sociales y humanas bajo el esquema del modelo universidadempresa-estado: una mirada desde la teoría crítica de la sociedad. Revistainteramericana desde la teoría Crítica de la sociedad. Medellín: Revista Interamericana de Bibliotecología. http://www.scielo.org.co/scielo.php?script=sci_arttext\&pid=S0120-09762009000200008

Mendidible Zurita, Alejandro. (2014). Brasil y su histórico viraje sudamericano, Caracas: Tiempo y Espacio 24(61), $79-103$.

Nayive Angulo Lilian. León R. Aníbal. (2005). Perspectivas Critica de Paulo Freire y su contribución a lateoría del currículo. Mérida. RevistaEducere. Recuperado de: http://ve.scielo.org/scielo.php?script=sci_arttext\&pid=S1316-49102005000200003

Nastidas de Figueroa Carmen. (2011). La Epistemología de la Complejidad en el desarrollo crítica de la humanidad. Caracas. Cuadernos del Cendes. Recuperado de: http://ve.scielo.org/scielo.php?script=sci_arttext\&pid=S1012-5082011000200006

Noguera José Antonio. (1996). La teoría crítica: de Frankfurt a Habermas una teoría de la acción comunicativa a la sociología. Barcelona: Universidad Autónoma de Barcelona. Departamento deSociología. Recuperado en: https://core.ac.uk/download/pdf/39002078.pdf

Ocampo López Javier. (2008). Paulo Freire y la Pedagogía del Oprimido. Boyacá. Colombia. Revista de la Historia de la educación latinoamericana. Universidad tecnológica y pedagógica de Colombia. Recuperado de: https://www.redalyc.org/pdf/869/86901005.

Regader Bertrand (2017). La teoría del aprendizaje de jean Piaget. Psicología y mente. Bogotá. https://psicologiaymente.com/desarrollo/teoria-del-aprendizaje-piaget

Romero José Manuel. Herbert Mercurse (2011). Los orígenes de la Teoría Crítica: contribuciones a una fenomenología del materialismo histórico, sobre filosofía concreta. Barcelona: Plaza y Valdés. https://dialnet.unirioja.es/servlet/articulo?codigo $=7258033$

Sánchez Cárdenas Rodrigo. (2008). La teoría Crítica de Max Horkeimer. Universidad Iberoamericana. México. D. f.: IBERO. http://ri.ibero.mx/handle/ibero/911

Sanfeliciano Alejandro: (2018). Los procesos de adaptación: la asimilación y la acomodación. Psicología educativa y de desarrollo. La mente es maravillosa. Bogotá.

Schmidt H. L (2017). Paradigmas del ser humano: aproximación al camino a la complejidad. Caracas. Revista Educación y Desarrollo Social.

Tomar Romero Francisca. (2013). El lugar del hombre y la Antropología en la Bioética. Murcia. España.Cuadernos de Bioética. Recuperado de https://www.redalyc.org/pdf/875/87528682004.pdf 